\title{
Ámbitos público y mediático en comunicación y salud
}

Recibido: 02 de diciembre de 2014 Aceptado: 20 de julio de 2015 Publicado: 30 de octubre de 2015
Mónica Petracci mpetracci@sociales.uba.ar

Universidad de Buenos Aires (Argentina)

Resumen: Comunicación y Salud es un campo de conocimiento que, actualmente, está en un proceso de crecimiento y consolidación. El propósito del artículo es analizar tres investigaciones cualitativas sobre articulaciones en comunicación y salud realizadas en Buenos Aires, Argentina: comunicar un mensaje de cuidado de la salud en un servicio de un hospital público, comunicar políticas públicas, comunicar noticias de salud en los medios de comunicación. Fueron aplicadas, respectivamente, entrevistas semiestructuradas a profesionales de la salud, tomadores de decisión, y periodistas. Los hallazgos muestran cómo el campo de la comunicación y salud contribuye a construir ciudadanía y participación social.

Palabras clave: Comunicación y salud, servicio de salud, profesionales de la salud, políticas públicas, tomadores de decisión, prensa gráfica, periodistas.

Abstract: Health Communication is a field of knowledge, which is in a growing and strengthening process at present. The aim of this article is to analyze three qualitative studies about communication and health articulations conducted in Buenos Aires, Argentina: to communicate a message of health promotion in a public hospital; to communicate public policies in the Ministry of Health, Argentina; and, finally, to communicate health news on the press. Semi-structured interviews were conducted with health care providers, decision makers and journalists, respectively. Findings show how Health Communication contributes to building citizenship and social participation.

Key words: Health Communication. Health Service, Health Care Providers, Public Policies, Decision Makers, Press, Journalists.

\section{Introducción}

El entramado sociocomunicacional de la salud, en especial de la salud pública, dio lugar, a partir de la segunda mitad del siglo pasado, a la formación de un campo de conocimiento 
(Beltrán, 2000; Casas y otros, 2004; Regis, 2007; Cuberli, 2008; Petracci y Waisbord, 2011; Cuberli y Soares de Araujo, 2014) que fue acompañado por documentos de conferencias internacionales cuyas definiciones sobre comunicación y salud ocupan un lugar de privilegio en el proceso de formación y tematización del recorrido (Alma Ata, 1978; Ottawa, 1986; Adelaida, 1988; Sundsvall, 1991; Jakarta, 1997; Ciudad de México, 2000; Bangkok, 2005; Nairobi, 2009; Helsinki, 2013). Una producción variada y profusa sobre temas, ámbitos comunicacionales y actores del proceso comunicativo (Petracci, 2012) ha sembrado el crecimiento de este campo de conocimiento.

La variedad temática refiere a las innumerables situaciones ligadas a la salud y la enfermedad que cuentan con reflexiones y acciones comunicacionales. Algunas de ellas son las siguientes: alcoholismo (Menéndez, 1990), cáncer (Orbell, 1996; Petracci, 2005a y 2009; Arrossi y Thouyaret, 2011), cólera (Petracci y otros, 1998; Petracci, 2002), cuidados (López y otros, 2012), dengue (Eynard y Drovetta, 2011), diabetes (Del Pozo, 2013), mortalidad materna (Ortiz y Ravalli, 2011), prematurez (Petracci, 2013), salud reproductiva (Pulerwitz y otros, 2012; Sengupta y Elias, 2012), síndrome urémico hemolítico (Belardo, 2011), tabaquismo (Mejía, 2011), VIH/sida (Pulerwitz y otros, 2012).

También la variedad aplica a ámbitos y actores del proceso comunicativo: la relación médicopaciente (Schvartz, 2011), las consultas en foros de salud en Internet (Alcántara López, 2015; March Cerdá, 2015), el vínculo entre profesionales de la salud en el equipo de un servicio así como entre ellos y el personal administrativo (Jalley y Goyos, 2011); la agenda pública y mediática (Gambaccini y otros, 2011; Jait, 2011; Kaufman, 2011), las campañas dirigidas a la ciudadanía en general o a sectores de ella, la construcción de noticias por el periodismo científico y en salud, las intervenciones comunitarias y el trabajo con organizaciones sociales, o bien las acciones de la industria farmacéutica (Vara, 2011), entre otros casos.

Sobre la pluralidad de objetos de estudio y la confluencia disciplinar de la comunicación y salud se asienta la riqueza analítica. En esa dirección, Petracci y Waisbord (2011: 9) sostienen que
"cualquier intento por capturar la diversidad de enfoques, modelos, y prácticas en comunicación y salud es necesariamente incompleto. Esto se debe a varios motivos. Los múltiples problemas sociales que atraviesan el campo, desde cuestiones de poder pasando por el espacio público y mediático, hasta la relación entre profesionales de la salud y usuarios de los servicios de salud. Los sesgos derivados de la formación de procedencia y las inquietudes personales volcadas sobre cualquier análisis".

Otra característica (previamente señalada) es el crecimiento de una producción profusa, que proviene de la descripción y la interpretación de resultados de intervenciones destinadas a la aplicación de una estrategia comunicacional y, en menor medida, de hallazgos de investigaciones empíricas y evaluaciones rigurosas. Lo anterior no es una crítica ni una originalidad. Por el contrario, con dicha afirmación se busca abrir, de par en par, una puerta a la reflexión teórica comunicacional desde la cual construir los problemas de investigación 
y analizar los hallazgos con el doble propósito de superar debates binarios y la tendencia a acumular, sin una sistematización académica rigurosa, resultados basados en la descripción de casos puntuales. Esta reflexión fue anudada con investigadores e investigadoras de distintas latitudes ${ }^{1}$ desde hace años:

"Aquí rescatamos una visión de la comunicación vinculada con el proceso de participación en la producción e intercambio de ideas y opiniones. No optamos por una visión ortodoxa 'informacional/cibernética' de la comunicación que equipara a esta última con la transmisión de información. Tal enfoque, ciertamente muy influyente en la tradición de estudios de efectos de medios desde planteos psicológicos y computacionales comunes en Estados Unidos, ofrece una perspectiva mecanicista y limitada que ignora complejos procesos sociales y políticos de intercambio y formación de sentido social como así también de participación política. Información no es comunicación; puede ser un componente no excluyente de procesos comunicacionales. Tampoco suscribimos a visiones de la comunicación que enfatizan sus raíces etimológicas en el sentido de la búsqueda de lo común y la comunidad. Tales objetivos pueden ser alcanzados pero no son determinados a priori en el proceso comunicacional. Son una posibilidad, no una necesidad, de la comunicación [...] Por estas razones, creemos que la comunicación, como disciplina, contribuye con el análisis de la salud como fenómeno social [...] Tales focos analíticos remiten a problemáticas que dominan el interés de las ciencias sociales como el poder, la cultura, el orden, el conflicto, el estigma y la discriminación, la formación de identidades entre otros. Tal enfoque es necesario para superar el foco 'campañista' de estudiar comunicación/salud principalmente en torno a intervenciones comunicacionales durante períodos determinados. Las campañas son una forma de intervención dentro de un contexto complejo y permanente de comunicación. No existen aisladas sino dentro de un conjunto de prácticas comunicacionales y conocimientos sobre salud a cuyo efecto sinérgico contribuyen" (Petracci y Waisbord, 2011: 13).

\section{Objetivo y metodología}

El objetivo general de este artículo es aportar evidencia sobre articulaciones entre comunicación y salud pública: el institucional, porque visibiliza los escenarios políticos y las tensiones propias de las relaciones de poder instituidas que atraviesan lo comunicacional; la política pública, porque muestra qué perspectivas teóricas comunicacionales tienen la formulación e implementación de planes y programas públicos; y los medios de comunicación, porque la construcción de noticias sobre salud permite entrever los circuitos comunicacionales entre periodistas y profesionales de la salud.

1. La autora de este artículo comparte esa reflexión con Inesita Soares de Araujo (Brasil), José del Pozo-Cruz (España), Silvio Waisbord (EE. UU.), Milca Cuberli y Andrea Palopoli (Argentina). 
Para el análisis de cada una de esas articulaciones se presentarán sendas investigaciones y objetivos. Metodológicamente se trata de tres estudios cualitativos cuyos lineamientos específicos se describen en cada sección.

\section{Hipótesis de trabajo}

A partir de la centralidad política y técnica de lo comunicacional en la sociedad contemporánea, los resultados (tanto los provenientes de la práctica investigativa como de la presencia de profesionales formados en la comunicación social en ámbitos institucionales gubernamentales y no gubernamentales) de los trabajos producidos en el campo comunicación y salud contribuyen con el tratamiento interdisciplinar.

De acuerdo con la caracterización enunciada sobre la construcción del campo, los interrogantes para abordar las articulaciones entre comunicación y salud apuntan a temas, actores y ámbitos de la comunicación, o a todos ellos conjuntamente, en la salud pública. Dicha afirmación se sostiene en una hipótesis general de trabajo, orientadora de la comprensión del problema investigado en tres estudios cualitativos que se exponen en la sección siguiente, que entiende el proceso comunicativo como una instancia participativa, de construcción de ciudadanía y apropiación de derechos, superador de la mera producción de contenidos para campañas destinadas a provocar cambios en los comportamientos individuales.

\section{Comunicación y Salud en el ámbito institucional: un hospital público}

El diseño, la puesta en marcha y los resultados alcanzados por una experiencia comunicacional están íntimamente conectados con el escenario político e institucional (Petracci y otros, 1998) en cuyo marco se toman las decisiones de diseño e implementación de la misma. En una institución de salud, lo comunicacional está presente en la relación médico-paciente, en el contenido de la comunicación sobre prevención, en la comunicación en el interior de un equipo de salud, o en los efectos de la inclusión de un profesional de la comunicación.

A continuación se presenta el desarrollo de una experiencia comunicacional cuyo objetivo fue promover la consulta en un servicio de salud de un hospital público ${ }^{2}$ (Petracci, 2009). A partir de reflexiones sobre contenidos de las campañas de interés público (Petracci, 1992), el aporte de la comunicación para el cambio social (Gumucio-Dagron, 2002) y de las perspectivas de género y derechos, específicamente el trabajo de investigación y comunicación estuvo destinado a promover la consulta al ginecólogo y los beneficios de la prueba de Papanicolau (una prueba sencilla cuya correcta toma en la frecuencia adecuada

\footnotetext{
2. Los hallazgos corresponden a la investigación "Cáncer cervical: calidad de la atención ofrecida por el equipo de salud y prevención a través de los medios de comunicación”, de la cual fui Directora. Sede: Centro de Estudios de Estado y Sociedad (CEDES). Financiamiento: PATH through GATES 2003-2004.
} 
puede salvar vidas de mujeres) en un servicio de salud de un hospital público emplazado en una zona de escasos recursos económicos del Partido de General San Martín, provincia de Buenos Aires, Argentina. El equipo de salud del servicio estaba preocupado porque había percibido (después de la crisis económica que afectara al país en 2001) que cada vez eran más las mujeres que, cuando acudían a atenderse, recibían un diagnóstico de cáncer avanzado.

El punto de partida del diseño comunicacional fue una investigación que tuvo por objetivo producir un diagnóstico cualitativo de la opinión del equipo de salud sobre la calidad de la atención, las necesidades comunicacionales y, finalmente, la proyección del resultado que imaginaban obtener como resultado de esa experiencia participativa. La técnica de relevamiento fue la entrevista grupal (Merton, 1987) con consentimiento informado. De dichas entrevistas con el equipo de salud para elaborar el diagnóstico surgió la idea de una pieza comunicativa (una tarjeta o carné e $^{3}$ ) destinada al cuidado de la salud ginecológica, y elaborada por sus integrantes en lo que hace a formato, colores, idea, etc., que el médico entregaría a las usuarias cuando concurrieran a atenderse de manera tal que esa información funcionara como un recordatorio de la visita siguiente. Se descartó el diseño de un folleto o de otro tipo de material de prevención destinado únicamente a mostrar los beneficios del Papanicolau. Cabe señalar que el equipo de salud no sólo manifestó una buena recepción de la idea sino que demostró una notable dedicación para presentar una versión de la pieza que quería que fuese el producto final.

El diagnóstico cualitativo demostró que los profesionales del equipo estaban interesados en disponer de una pieza que instalara en las usuarias un comportamiento de cuidado de la salud ginecológica, como parte de un proceso de mejora de la calidad de la atención. Las ideas fuerza fueron la recordación del Pap y la visita al ginecólogo. El supuesto básico de ambos mensajes era que la recordación del Pap, unida a la fecha de la próxima visita, en una misma pieza, promovería en la usuaria una mayor propensión a sostener y no discontinuar las consultas. La función secundaria del carné, en el marco de las perspectivas de género y derechos sexuales y reproductivos, era brindar información sobre la prueba de Pap y la colposcopía, la necesidad de otro estudio como la mamografía, la prevención de Infecciones de Transmisión Sexual y de VIH/sida. El contrato de lectura, a través de un lenguaje coloquial, apuntó a generar en la usuaria la percepción de que el profesional era quién había escrito ese mensaje para ella y, a su vez, promover su autonomía y apropiación de derechos, aspectos considerados decisivos para propiciar el cuidado de su salud. Dicha pieza comunicacional fue acompañada con una guía de recomendaciones para la presentación destinada al equipo de salud.

En cuanto a las fortalezas de la experiencia comunicativa, en primer lugar cabe destacar que fue el resultado de una investigación empírica, en este caso de tipo cualitativo. La perspectiva relacional adoptada favoreció la reflexión y el intercambio dentro del equipo de salud a través del proceso de imaginar colectivamente todos los aspectos de la pieza

3. Véase una imagen del carné en Petracci (2009). 
comunicacional para promover en las mujeres la consulta ginecológica regular. También fue una fortaleza el proceso a través del cual el equipo de salud tomó conciencia de su rol de comunicadores ante la presentación del carné a las usuarias y, por sobre todo, de visibilización de su responsabilidad personal e institucional en la promoción de prácticas de cuidado más que en la focalización de la prevención como comportamiento individual. Tal como señala Douglas (1996), al referirse a la construcción social del riesgo, la prevención es una decisión social que necesita ser explicitada por las instituciones.

Entre las debilidades puede mencionarse la falta de armado de redes, si bien fue considerada la difusión del carné a otras mujeres. El trabajo empezó y terminó con los integrantes del equipo de salud del servicio sin la participación activa de las usuarias, quienes podrían haber armado redes favorecedoras del intercambio de mensajes entre las mujeres. Cabe señalar que la opinión de las usuarias fue tenida en cuenta en la etapa correspondiente a la investigación pero, por los motivos de diversa índole que inciden en la realización de experiencias y proyectos y que exceden el alcance de este artículo, no se incluyó en el diseño y la primera aplicación del carné. Otra debilidad fue la ausencia de evaluaciones para observar el comportamiento del carné una vez instalado.

Desde el lado de las usuarias del servicio/destinatarias del mensaje, hubiera sido conveniente realizar un estudio cualitativo de recepción de mensajes a corto plazo y un estudio de medición del impacto del carné en la asistencia a la consulta a mediano y largo plazo. Desde el lado del equipo de salud, también hubiera sido conveniente realizar un estudio para observar los beneficios y las dificultades del carné de manera tal que futuras versiones de esa pieza comunicacional hubieran podido ser mejoradas tomando en cuenta la perspectiva de los actores intervinientes. Otro aspecto a considerar hubiera sido la inclusión del personal administrativo y su entrenamiento en habilidades comunicacionales para reforzar el uso del carné.

La creación de una pieza comunicacional (el carné), en el marco de un servicio de salud de un hospital público, fue una etapa colmada de fortalezas y de buenas intenciones de los actores. No obstante, la aplicación estuvo atravesada por los conflictos y las tensiones propios de las relaciones de poder instituidas. La principal debilidad, que en general afecta a todo cambio y en especial a la comunicación social, estuvo dada por la dificultad para construir una cultura institucional de cuidado, de la cual la pieza producida fuera sólo una parte. Son las instituciones y el Estado, más que los sujetos, los encargados de construir una cultura de la prevención y el cuidado al definir estratégicamente el rol de la comunicación en las instituciones de salud. 


\section{Comunicación y salud en el ámbito público estatal: las perspectivas comunicacionales de tomadores de decisión de políticas públicas ${ }^{4}$}

Las comunicaciones que emanan de las instituciones gubernamentales conforman un conjunto de discursos sociales prácticamente imposibles de soslayar para comprender la actividad esencial del Estado: la formulación e implementación de políticas públicas. Los escenarios políticos, las perspectivas y las capacidades de comunicación consolidan el accionar de los actores clave en la toma de decisiones sobre lo público y las políticas públicas, entendidas siguiendo a Oszlak (1984: 5), como "un conjunto de sucesivas tomas de posición [...] frente a cuestiones socialmente problematizadas".

Dicho en otros términos: las políticas públicas son decisiones sobre prioridades, sobre los objetivos y las acciones para cumplimentarlas. Lo comunicacional es constitutivo de una política pública, de salud en este caso, en cualquiera de sus etapas, desde la formulación hasta la implementación a los destinatarios o la instalación en la agenda pública. En esa dirección, la bibliografía ha estudiado los discursos sociales de las políticas públicas en tanto y en cuanto los mismos se reflejan en los medios de comunicación, o bien los motivos que convierten a un tema en una cuestión socialmente problematizada a tratar por el Estado y los aspectos comunicacionales correspondientes.

En síntesis, la distinción entre lo "político" y lo "burocrático" (implícita) en las definiciones previas, por discutible que ella pueda resultar, representa un punto de partida para el análisis de los discursos sociales de las políticas públicas. Aunque pueda ser inválida en un examen en profundidad, ambas categorías existen fenomenológicamente como "ámbitos finitos de sentido", aceptados como tales por el grueso de los actores sociales. Todo ello es comunicación y requiere ser estudiado en su pragmática, conformando un material de análisis que da cuenta de las particularidades que reviste el proceso comunicativo emanado de las agencias gubernamentales tanto con respecto a una política sectorial como al estilo comunicacional de un funcionario.

En el estudio que se presenta a continuación, a diferencia, se plantearon interrogantes sobre las perspectivas comunicacionales adoptadas por los tomadores de decisión en el marco del debate entre las perspectivas comunicacionales "difusionista" y "procesual", cuyas diferencias son planteadas extensamente por la literatura. La primera valora la información, los soportes mediáticos, y sostiene una visión lineal del proceso comunicativo. La segunda valora la participación, la incorporación transversal y colectiva de la comunicación a los procesos de cambio social, los medios comunitarios, acentúa la complejidad del proceso comunicativo, la participación de los destinatarios, y las mediaciones sociales. Sobre esa distinción, Díaz (2011: 41) resume con claridad:

4. Corresponde a uno de los objetivos del proyecto UBACyT 2011-14: "La salud en la trama comunicacional contemporánea”, dirigido por M. Petracci. Integraron el equipo: J. Brown, L. Carral, M. Cuberli, A. Gurfein, M. Mattioli, A. Palopoli, C. Pardo, P. Rodríguez Zoya y C. Straw. 
"Si la perspectiva instrumental se basaba en el poder de los emisores como difusores de información en la creencia de que 'los destinatarios son estúpidos', la propuesta relacional invierte esta visión y reprocha a quienes sostienen esas teorías el no pensar en la recepción como la instancia fundamental del proceso. 'Son los destinatarios, estúpidos', les dice a los teóricos de aquella visión. ¿Quiere decir esto que todos podemos resignificar libremente los mensajes sin ningún tipo de condicionante? Por supuesto que no, porque es de reconocer que no todos ocupamos el mismo lugar en una sociedad y que hay determinados discursos y sentidos que tienen una mayor capacidad para ser escuchados, leídos o percibidos. Lo que esta perspectiva hace es incorporar dos elementos clave que permiten entender mejor lo que sucede en los fenómenos sociales en tanto procesos de producción de sentido: las mediaciones a través de las cuales se construyen las relaciones sociales y la cultura como terreno que abona (y condiciona) estas relaciones".

Dos hipótesis guiaron la investigación: a) las perspectivas comunicacionales de las políticas públicas en salud guardan relación con los escenarios políticos de cada política pública (Petracci, Quevedo y Vacchieri, 1998) y b) la perspectiva 'difusionista' tiene más peso que la 'procesual relacional' en las estrategias comunicacionales públicas. El objetivo fue explorar las perspectivas comunicacionales de los tomadores de decisión de los programas y planes del Ministerio de Salud de Nación, Argentina.

La exploración de las perspectivas comunicacionales fue realizada de manera indirecta a través del conocimiento de las comunicaciones realizadas, las opiniones sobre el diseño de las piezas, y la importancia asignada a lo comunicacional, entre otros temas. Metodológicamente se realizó un estudio exploratorio, cualitativo, mediante entrevistas con consentimiento informado a tomadores de decisión (n: 17), responsables de los programas. El proceso de análisis destinado a asignar una perspectiva comunicacional a cada entrevista, realizado por el equipo a través de consensos sucesivos, fue tan arduo como interesante.

Del análisis se desprende, en primer lugar, que los tomadores de decisión aceptan la dimensión comunicacional en sus programas. En esta aceptación inciden la creación de una dependencia específica, la Coordinación Nacional de Información Pública y Comunicación (CYPyC) del Ministerio de Salud de la Nación y la presencia de profesionales egresados de Carreras de Comunicación, especialmente de la Universidad de Buenos Aires, en Programas y Planes. Uno de los logros de esa institucionalización fue la realización de una encuesta a comunicadores integrantes de áreas, direcciones y programas del Ministerio de Salud de la Nación y sus pares provinciales que dio como resultado el primer Mapeo de Capacidades en Comunicación y Salud (Bruno y otros, 2011).

No obstante la decisión política y la consolidación disciplinar, la aceptación de los tomadores de decisión ancla en una visión instrumental de lo comunicacional que, como se sostiene en la primera hipótesis, se plantea atendiendo a los actores sociales partícipes 
del escenario político de cada política sectorial. En segundo lugar, los hallazgos evidencian que, a diferencia de lo sostenido en la segunda hipótesis, lejos de encontrar extremos informativistas o procesuales "puros", ambas perspectivas consideradas coexisten.

\section{Comunicación y salud en los medios de comunicación: la construcción de las noticias de salud}

Las noticias sobre salud han registrado un crecimiento considerable en los medios de comunicación en los últimos años, a través de la creación de secciones especiales y publicaciones especializadas dedicadas a brindar información sobre salud, prevención, enfermedades, evidencias, entre otros. ¿Qué condicionantes y tensiones existen entre la construcción de la noticia periodística (Colombo, 1997; Martini, 2000) en salud, en el espacio mediático y las expectativas de los profesionales?, ¿puede el discurso periodístico construir un lenguaje común entre profesionales de la salud y de los medios?

Las características y las controversias, planteadas alrededor de la cobertura de noticias sobre salud en los medios de comunicación, nos llevaron a profundizar una perspectiva menos explorada en la literatura, la de los periodistas argentinos especializados en salud, con trabajo en medios gráficos, respecto de los atributos de noticiabilidad más empleados en esta rama del periodismo. Dado que periodista y fuente de información pueden tener diferentes conceptos sobre qué es una noticia, la hipótesis de este trabajo sostiene que los lenguajes científico-médico y periodístico entran en colisión frente a los atributos de noticiabilidad (Loewy, 2010).

Esos criterios que guían la selección de notas a ser publicadas o difundidas, derivadas de la práctica periodística o la cultura organizacional de cada medio, no siempre son compartidos por los profesionales de la salud, quienes habitualmente preferirían que al "valor sanitario" de la información se le otorgase más relevancia, por encima de la fría ponderación de su valor noticioso (Loewy y Petracci, 2015).

El objetivo de este estudio 5 ( $c f$. Loewy y Petracci, 2015) fue explorar y describir las opiniones y las experiencias de periodistas de medios de referencia nacional en el proceso de construcción de la/s noticia/s en salud en la agenda pública y mediática ${ }^{6}$. Se toma la definición de noticia de Alsina (1993: 18), una representación social de la realidad cotidiana, institucionalmente producida, y contribuye a la construcción de un mundo posible, que incluye tres dimensiones: a) la construcción de la noticia a partir de los acontecimientos que diariamente se seleccionan; b) la institucionalización, es decir la noticia se produce en una institución informativa que supone una organización compleja; y c) la noticia es una producción discursiva y tiene, como tal, un proceso de elaboración textual.

5. Corresponde a uno de los objetivos del proyecto UBACyT 2011-14 "La salud en la trama comunicacional contemporánea", dirigido por M. Petracci.

6. Cabe señalar que no se incluyó en este análisis a la noticia sobre salud con fines comerciales. 
Metodológicamente se realizó un estudio exploratorio, cualitativo, con entrevistas (con consentimiento informado) a diez periodistas dedicados en forma exclusiva o prioritaria a la cobertura de salud y/o ciencia en medios gráficos de Argentina. Se eligieron periodistas de medios gráficos porque, en la Argentina, dichos medios todavía tienen mayor capacidad de marcar agenda, orientar la cobertura de otros medios e influir en la tematización de las discusiones en la esfera pública. Con respecto a la selección de los entrevistados a ingresar a la muestra (no probabilística) se consideró la pertenencia a diferentes medios de distribución nacional y regional. Asimismo, se decidió no incluir en la muestra a aquellos médicos que se definen como "periodistas médicos" o "periodistas científicos", dado que sus percepciones y opiniones podrían haber quedado marcadas por los valores de su profesión de origen. Para definir la muestra se consideraron relevancia, propósito y saturación (Glasser y Strauss, 1967)

En cuanto a las características sociodemográficas, ocho periodistas son mujeres, dos son varones. Cinco trabajan en diarios de la Ciudad de Buenos Aires; uno, en un sitio web de salud cuyo contenido suele salir en un diario de la Ciudad de Buenos Aires; cuatro en diarios del interior del país: Comodoro Rivadavia, Córdoba, Rosario, y Salta. En cuanto al número de años de orientación prioritaria o exclusiva al periodismo en salud, el número de años mínimo es 3 y el máximo 30. Asimismo, dos de ellos escribían también para sitios web, seis tenían o habían tenido participación en programas de radio, y uno era columnista de salud en televisión.

A continuación se presentan los hallazgos sobre tres de los temas indagados: las opiniones sobre la responsabilidad profesional, la valoración de los atributos de noticiabilidad en salud, y las opiniones sobre el tipo de relación establecido entre periodistas y profesionales de la salud.

\subsection{Con respecto a la responsabilidad profesional}

Existe consenso en los periodistas entrevistados acerca de la responsabilidad social especial de su trabajo tanto por la naturaleza sensible de las temáticas que cubren como por el potencial impacto de esa información sobre el público. Pero también porque la práctica hace que no solamente informen y satisfagan las expectativas de sus medios sino que procuren educar a la población sobre salud y derechos, lo que, en ocasiones, los puede llevar a confrontar o negociar con aquellos editores concentrados en los valores periodísticos tradicionales sin tomar en cuenta el carácter singular de las noticias de salud.

Otro aspecto de la responsabilidad profesional está basado en la asunción de que, efectivamente, la información que transmiten tiene efecto sobre las personas. Al respecto, hay un consenso en las respuestas: la información no cambia conductas per se, sino que puede despertar o generar inquietud sobre determinada enfermedad, práctica preventiva o situación de salud, favoreciendo la consulta a los profesionales. 


\subsection{Sobre la valoración de los atributos de noticiabilidad en salud}

En lo referido a este punto, las opiniones se concentran y dispersan. La "extensión" o "magnitud" es el atributo con mayor consenso. Alude a la cantidad de personas afectadas por el acontecimiento, lo cual, en el campo de la medicina, orienta la cobertura de aquellas enfermedades o problemas de salud que tienen una mayor prevalencia. El atributo es reconocido como decisor en la lógica periodística pero también cuestionado. Para algunos periodistas entrevistados, esa valoración cuantitativa puede resultar una traba frustrante a la hora de impulsar la cobertura de patologías menos frecuentes. El atributo de magnitud o extensión es tan fuerte en el periodismo de salud que, en palabras de uno de los periodistas, las asociaciones de pacientes con enfermedades raras tienen que hacer un "lobby noble" para aumentar la visibilidad de esas patologías.

En segundo lugar, otros atributos de noticiabilidad señalados son la "novedad" y la "oportunidad". La novedad implica una ruptura actual o muy reciente de lo cotidiano. Se considera que lo urgente, lo inmediato, impacta más que la información ya conocida o que se conoce mucho tiempo después de que algo haya ocurrido. También se señaló que si bien la novedad era un criterio tradicional del periodismo para construir una noticia, en la práctica actual cumple la función de "gatillo" o excusa para exponer un tema que, aunque no sea novedoso, involucra al público al cual se dirige el medio. También el criterio difiere según se publique en la tapa o en un suplemento temático.

Es interesante destacar el consenso alcanzado en el segundo lugar asignado a la "novedad" como atributo de noticiabilidad. El hecho de que los periodistas de salud entrevistados hayan relegado el atributo de actualidad, cuando para la mayor parte de los periodistas de otras ramas representa el valor noticioso más relevante (una de las definiciones de noticia es "hecho actual o reciente"), podría derivar de la caracterización de la salud como una sección "fría" en la prensa, ajena al arrebato en la redacción característico de otras temáticas. Como puntualiza Waisbord (2010: 186-187):

“La salud es usualmente vista como 'información de parrilla' [...] desligada de la 'noticia caliente' que típicamente impulsa el vértigo diario en las redacciones. Esto se debe, en parte, a que la noticiabilidad de los temas de salud no es perecedera. La información no está sujeta al paso rápido del tiempo, que hoy en día, debido a la aceleración de la producción de la noticia, se calcula en horas o minutos. La relevancia temporal de información sobre nuevos tratamientos quirúrgicos, índices de mortalidad materna, o tendencias en consumo de drogas no es fungible como lo es la información que se considera "urgente",.

La oportunidad alude a aquella información que se relaciona con un tema que está en la agenda de los medios y por ende en la preocupación colectiva. Un caso emblemático serían las historias con foco en determinada patología que acaba de ser diagnosticada en un famoso. O aquellas notas que se publican "a propósito de" una fecha o aniversario, por ejemplo, el día internacional de lucha contra tal o cual enfermedad, o cuando se cumplen 
los treinta años de una primera operación considerada exitosa. Se percibe que ese mismo contenido informativo, en otro momento, no tendría tanto atractivo para el lector. Ni para el editor. De las entrevistas se desprende que, en muchos casos, el atributo de oportunidad fue considerado por los participantes, precisamente, como una excelente ocasión para hacer promoción de la salud sin vulnerar la lógica y las reglas de la práctica periodística. En ese sentido, ciertas novedades, como el lanzamiento de una campaña de concientización, suele ser aprovechada para profundizar en esa temática. El criterio de oportunidad parece ser, quizás, el que mejor reconcilia el rol del periodista como productor de noticias y su aspiración como agente educativo en salud.

La "importancia" del tema, en tercer lugar, fue mencionada como valor noticia. Cabe aclarar que este atributo puede solaparse con el atributo de extensión. En los manuales de periodismo, la importancia o impacto social hace referencia a aquellos acontecimientos con efectos, influencias, consecuencias o implicaciones para la sociedad o amplios sectores sociales. También es importante enfatizar, de todos modos, que la importancia, como otros atributos de noticiabilidad, está sujeta a la subjetividad del periodista o del medio. ¿Un avance experimental contra el cáncer que recién podría tener aplicación clínica en 20 años, es importante o no? Lo que puede ser relevante para un medio puede no serlo para un médico y viceversa.

Con una importancia menor fueron mencionados los siguientes atributos: la "utilidad" o servicio, la "proximidad", la "positividad" y la "rareza". El primero refiere a aquellas noticias que ofrecen información práctica para el lector (consejos, recomendaciones preventivas, sitios, etc.), consideradas "de servicio" por los periodistas. Si bien pueden no tener novedad alguna, son, quizás, aquellas que los médicos pretenderían ver publicadas más a menudo en los medios. La proximidad, mencionada por periodistas del interior del país, refiere a un hecho cercano cultural o territorialmente al lector es más susceptible de transformarse en noticia que otro alejado de sus intereses o preocupaciones. La positividad refiere a la percepción de que las noticias "felices" sobre curas o nuevos tratamientos tienen más chance de encontrar lugar en la pauta que aquellas historias sobre, por ejemplo, las vivencias cotidianas de pacientes con enfermedades crónicas. La rareza refiere al carácter atípico o extraordinario de un acontecimiento.

Los atributos no son fijos ni compartimentos estancos. Son, en términos de Wolf (2004), el conjunto de requisitos que se le exige a los acontecimientos para adquirir la existencia pública de noticias. Hoy por hoy reciben las influencias de complejos escenarios mediáticos en los que intervienen. Las referencias a las consultoras en salud y las necesidades del público muestran cambios en la "novedad", un atributo considerablemente valorado por los periodistas entrevistados.

\subsection{Criterios de noticiabilidad empleados}

Respecto de las opiniones sobre el tipo de relación entre los criterios de noticiabilidad empleados por los medios y las expectativas de los profesionales de la salud, las respuestas exhiben consensos y disensos. Quienes señalaron que la relación es "armónica" señalaron 
que consensuaban con los profesionales aquellos temas que merecían una mayor cobertura en los medios o hasta afirmaron que los médicos más hábiles conocían a menudo los criterios de noticiabilidad periodísticos (como la novedad y la magnitud), al punto de reconocer que determinada información podría no tener cabida en los medios pese a que ellos la consideraran importante.

En cambio, otros entrevistados aludieron espontáneamente a las clásicas tensiones culturales ya descritas entre médicos y periodistas, como, por ejemplo, la exigencia de los profesionales de leer las notas antes de su publicación, la reticencia a dar notas por malas experiencias anteriores o la crítica por la falta de rigor de los textos periodísticos. Y otros, en particular, deslizaron la dificultad de los médicos para entender la importancia periodística de que exista un hecho noticioso concreto como "disparador" para poder transmitir una información de salud. O bien señalaron la desvalorización del trabajo periodístico, el desconocimiento o la falta de confianza.

De los hallazgos previos se desprende que la discordancia en términos de lo que resulta "noticiable" o no, se enmarca en una serie de tensiones culturales, bien descriptas en la literatura, entre médicos y periodistas (Nelkin, 1996; Ransohoff y Ransohoff, 2001; Loewy, 2010). En términos generales, los periodistas de salud entrevistados en este estudio perciben que existe a priori una desconfianza de los médicos respecto de su capacidad para interpretar y transmitir de manera rigurosa el discurso médico. También los médicos pueden sospechar que la selección de determinado tema o fuente está guiada por intereses comerciales y no por legítimos valores periodísticos. O que existe una tendencia al sensacionalismo.

Sin embargo, esa desconfianza, originada a menudo en "malas experiencias" anteriores, puede atenuarse o disiparse a medida que los médicos conocen la trayectoria del periodista o cuando lo ven desenvolverse con conocimiento del tema durante la entrevista. Loewy (2010: 157) argumentaba que:

"el tamiz de los criterios de noticiabilidad, extendido en la rutina productiva de los medios, desecha temas que los médicos podrían considerar de importancia central para la salud pública; y en cambio, retiene, procesa y alienta la transformación en noticias de otras informaciones que pueden tener menor trascendencia o impacto epidemiológico, pero que reúne ciertos rasgos que le permiten competir de forma digna en la puja por el espacio o el aire con otras noticias que en principio tratan sobre temas más atractivos, como las políticas, policiales o deportivas. El periodista de salud no 'sucumbe' a la ponderación de los criterios de noticiabilidad porque sea insensible a otras dimensiones de la información que maneja, sino porque suele estar obligado a usar las mismas reglas de juego de sus colegas que cubren otras secciones o temas en el mismo medio".

Desde esa óptica, se deslizaba que uno de los puntos de desencuentro entre periodistas y médicos se vincula a aquellas situaciones en que la "finalidad sanitaria" de la información 
de salud, esperada por los médicos, entra en colisión con la ponderación de la calidad de la noticia bajo criterios estrictamente periodísticos, por ejemplo, los tipos y la cantidad de atributos de noticiabilidad satisfechos o incluso su veracidad o equilibrio.

No caben dudas que los señalamientos de los periodistas de salud entrevistados enfatizan la variedad, la complejidad y los cambios en los valores de noticiabilidad. Según Román (2011: 175) "varían según los medios para los cuales cada periodista trabaje y son bastante dinámicos. El tamiz por el que pasan las informaciones es tan complejo y a veces hasta desconocido por los mismos periodistas que elaboran la noticia final". Tampoco caben dudas que desde sus perspectivas, los criterios de noticiabillidad pueden entrar en colisión para profesionales médicos y periodistas especializados. Mientras no exista una mayor comprensión y sensibilidad mutuas respecto de los condicionamientos productivos y culturales de unos y otros, una construcción compartida de la noticia en salud está lejos de ser posible.

\section{Conclusiones}

En este artículo se presentaron los hallazgos de tres investigaciones sobre articulaciones en comunicación y salud: comunicar un mensaje de cuidado de la salud en un servicio de un hospital público, comunicar políticas públicas y comunicar noticias de salud en los medios de comunicación. Los problemas de investigación y los estados del arte, fueron construidos desde interrogantes sociales (especialmente comunicacionales) y la información fue relevada en aquellos actores sociales menos indagados por la literatura: el equipo de salud, los tomadores de decisión y los periodistas en salud, respectivamente.

La construcción institucional del cuidado y la prevención en un marco de relaciones de poder instituidas. La superación del debate binario, en el sentido de que el debate se constituya como pensamiento binario y los actores sostengan, en la práctica, una confrontación que vuelve estéril el debate y lo torna, además, obsoleto para comprender y explicar fenómenos sociales complejos. La dificultad de encuentro entre los lenguajes periodístico y científico en el marco de la construcción de la noticia de los medios de comunicación... son articulaciones que muestran la diversidad y la riqueza del campo comunicacional de la salud. Plantean líneas de investigación a conformar y dan lugar a formular nuevas preguntas temáticas y metodológicas: ¿qué opinan los destinatarios de los mensajes?, ¿cuál y cómo es la evaluación de una experiencia comunicacional?, ¿qué sucede con las noticias sobre salud en los medios masivos frente a la expansión de las redes sociales?, ¿aplican los resultados de un estudio a nivel nacional en el nivel local?

No obstante lo realizado, el trabajo a llevar adelante en el futuro es el planteo de las temáticas sociales en las cuales estén insertos los problemas y situaciones de salud a resolver comunicacionalmente (riesgo, medicalización, técnica, etc.); la sistematización de las investigaciones empíricas y las experiencias ya realizadas; la construcción de conocimiento que incluya movilización social, acciones de incidencia en políticas públicas, democratización de las relaciones sociales. Y así este campo joven estará a las puertas de su madurez. 
Finalmente, consolidar este campo significa también implicaciones positivas para la democracia en el sentido de que cada ciudadano goce de los derechos de la comunicación y su relación con los derechos humanos básicos civiles, políticos, económicos, sociales y culturales.

\section{Fuentes consultadas}

Alcántara López, R. (2015). “Comunicación \& Internet \& salud: puntas de un iceberg llamado e-salud”, pp. 45-58. En Del Pozo Cruz, J. y otros. Medios de Comunicación y Salud. Sevilla. Editorial Astigi.

Arrossi, S. y Thouyaret, L. (2011). "La comunicación en una estrategia integral para mejorar la participación de las mujeres en los programas de tamizaje de cáncer cérvicouterino", pp. 375-398. En Petracci, M. y Waisbord, S. (comps.). Comunicación y salud en la Argentina. Buenos Aires: La Crujía Ediciones.

Belardo, M. (2011). "Enfermedad transmitida por alimentos. El Síndrome Urémico Hemolítico y su inclusión en las agendas biomédica, social y política", pp. 421-438. En Petracci, M. y Waisbord, S. (comps.). Comunicación y salud en la Argentina. Buenos Aires: La Crujía Ediciones.

Beltrán, L. (2000). Investigación sobre comunicación en Latinoamérica: inicio, trascendencia y proyección. La Paz: Plural.

Bruno, D. y otros (2011). "Mapeo nacional de capacidades en comunicación y salud en Argentina”. Revista de Comunicación y Salud. Vol. 1, núm. 2, pp. 5-18.

Casas, L.; Cuberli, M. y Demonte, F. (2004). "La comunicación en el ámbito de la salud: perspectivas para repensarla”. Salud y Población, núm. 4. Extraída el 2/IX/2015 desde http://10.10.1.83/areas/salud/dircap/mat/rrieps.pdf

Colombo, F. (1997). Últimas noticias sobre el periodismo. Barcelona: Anagrama.

Cuberli, M. (2008). "Perspectivas comunicacionales para pensar las prácticas en salud: pasado y presente de un campo en construcción". Revista Question. Vol. 8, núm. 18. Extraída el 2/IX/2015 desde http://perio.unlp.edu.ar/ojs/index.php/question/article/view/524/443

Cuberli, M. y Soares de Araujo, I. (2014). "Las prácticas de la comunicación y salud: intersecciones e intersticios”. En Petracci, M. (2015). La salud en la trama comunicacional contemporánea [en prensa]. Buenos Aires: Prometeo.

Del Pozo, J. (2013). "Estrategias de comunicación para la prevención y promoción de la salud en pacientes con diabetes tipo 2 en la provincia de Sevilla" [tesis doctoral]. Sevilla: Universidad de Sevilla, Facultad de Comunicación. 
Díaz, H. (2011). "La comunicación para la salud desde una perspectiva relacional”, pp. 33-50. En Cuesta, U.; Menéndez, T. y Ugarte, A. (coords.). Comunicación y salud. Nuevos escenarios y tendencias. Madrid: Editorial Complutense.

Diez, P. y otros (2011). "Buenos Aires Salud 2003: una experiencia innovadora de comunicación en salud", pp. 227-248. En Petracci, M. y Waisbord, S. (comps.). Comunicación y salud en la Argentina. Buenos Aires: La Crujía Ediciones.

Douglas, M. (1996). La aceptabilidad del riesgo según las ciencias sociales. Barcelona: Paidós Ibérica.

Eynard, M. y Drovetta, R. (2011). "El vector subversivo: la epidemia de dengue en abril de 2009 en la ciudad de Córdoba y su construcción desde la prensa escrita local”, pp. 89-116. En Petracci, M. y Waisbord, S. (comps.). Comunicación y salud en la Argentina. Buenos Aires: La Crujía Ediciones.

Gambaccini, A.; Ramírez Barahona, P. y Salerno, V. (2011). "La salud que muestra la TV", pp. 65-88. En Petracci, M. y Waisbord, S. (comps.). Comunicación y salud en la Argentina. Buenos Aires: La Crujía Ediciones.

Glasser, B. y Strauss, A. (1967). The Discovery of Grounded: Strategies for Qualitative Research. Chicago: Aldine.

González Gartland, G. (2011). "La comunicación en la implementación de políticas de salud: el caso del Programa Remediar", pp. 399-420. En Petracci, M. y Waisbord, S. (comps.). Comunicación y salud en la Argentina. Buenos Aires: La Crujía Ediciones.

Gumucio-Dagron, A. (2002). "Haciendo olas: comunicación participativa para el cambio social”. Extraída el 24/VIII/2015 desde http://www.comminit.com/es/node/150011

Jait, A. (2011). "Los 100 días que vivimos en peligro: la construcción del nuevo virus de la influenza (H1N1) en Clarín”, pp. 117-142. En Petracci, M. y Waisbord, S. (comps.). Comunicación y salud en la Argentina. Buenos Aires: La Crujía Ediciones.

Jalley, V. y Goyos, E. (2011): “La comunicación interna en la gestión institucional: avatares y desafíos", pp. 249-268. En Petracci, M. y Waisbord, S. (comps.). Comunicación y salud en la Argentina. Buenos Aires: La Crujía Ediciones.

Kaufman, A. (2011). "Hacia una epidemiología del pánico mediático", pp. 143-152. En Petracci, M. y Waisbord, S. (comps.). Comunicación y salud en la Argentina. Buenos Aires: La Crujía Ediciones.

Loewy, M. (2010). “¿Lo importante es la salud? Condicionantes, limitaciones y tensiones culturales alrededor de las noticias médicas", pp. 153-170. En Petracci, M. y Waisbord, S. (comps.). Comunicación y salud en la Argentina. Buenos Aires: La Crujía Ediciones. 
Loewy, M. y Petracci, M. (2015). "Salud y noticiabilidad: las perspectivas de periodistas de medios gráficos de Argentina”, pp. 81-110. En Del Pozo Cruz, J. y otros (2015). Medios de Comunicación y Salud. Sevilla. Editorial Astigi.

López, E. y otros (2012). “Quereres y deberes: ¿cómo cuidan las mujeres su salud y la de su familia?”. En Kornblit, A.; Camarotti, A. y Wald, G. (comps.). Salud, sociedad y derechos: investigaciones y debates interdisciplinarios. Buenos Aires: Editorial Teseo. Extraída el 2/IX/2015 desde https://www.teseopress.com/saludsociedadyderechos/chapter/quereres-ydeberes-como-cuidan-las-mujeres-su-salud-y-la-de-su-familia/

Lucero, S. (2005). Las políticas de comunicación de las organizaciones públicas, un modelo de construcción identitaria desde los medios de comunicación [tesis de licenciatura]. Buenos Aires: Universidad Nacional de General Sarmiento, Instituto del Desarrollo Humano.

Madeira da Silva, W. (2011). Transformar é Preciso: transformacoes na relacao de poder estabelecida entre médico e paciente: um estudo em comunidades virtuais [tesis doctoral]. San Pablo: Universidad de San Pablo.

March Cerdá, J. (2015). “Redes sociales, salud y pacientes”, pp. 181-226. En Del Pozo, J. y otros. Medios de Comunicación y Salud. Sevilla: Editorial Astigi.

Martini, S. (2000). Periodismo, noticia y noticiabilidad. Buenos Aires: Grupo Editorial Norma.

Mejía, R. (2011). "Los medios de comunicación en la prevención y el control del consumo de tabaco", pp. 355-374. En Petracci, M. y Waisbord, S. (comps.). Comunicación y salud en la Argentina. Buenos Aires: La Crujía Ediciones.

Menéndez, E. (1990). Morir de alcohol: saber y hegemonía médica. México D. F.: Alianza Mexicana.

Merton, R. (1987). “The focussed interview and focus groups". Public Opinion Quarterly, vol. 51, pp. 550-566.

Nelkin, D. (1996): "Uneasy relationship: the tensions between medicine and the media". Lancet 347:1600-3

Orbell, S. (1996). "Cognition and affect after cervical screening: the role of previous test outcome and personal obligation in future uptake expectations". Social Science and Medicine. Vol. 43, núm. 8, pp. 1237-1243.

Oszlak, O. (1984). Politicas públicas y regímenes políticos. Buenos Aires: Estudios CEDES. 
Ortiz, Z. y Ravalli, M. (2011). “Desafíos en la comunicación de las muertes maternas", pp. 321-334. En Petracci, M. y Waisbord, S. (comps.). Comunicación y salud en la Argentina. Buenos Aires: La Crujía Ediciones.

Petracci, M.

_(2013). "Semana del Prematuro UNICEF. Estrategia comunicacional. Informe final". Buenos Aires: UNICEF.

_(2012). "Comunicación y salud: un campo diverso y pujante". Organicom, Revista Brasileira de Comunicacao Organizacional e Relacoes Publicas. Año 9, núms. 16/17, pp. 40-49.

_(2009). “Comunicación estratégica y salud: reflexiones sobre la experiencia de promover el papanicolau (pap) en un hospital público del Gran Buenos Aires, Argentina”. Revista Question, núm. 21. Extraída el 2/IX/2015 desde http://perio.unlp.edu.ar/ojs/index.php/ question/search /search

_(2005a). "Cáncer cervical: prevención a través del equipo de salud y los medios de comunicación", pp. 75-104. Concurso Haydeé de Luca 2005 del Comité Argentino de Educación para la Salud (CAESPO).

_(2005b). "La perspectiva de los profesionales de la salud sobre la calidad percibida por los usuarios/as: dos opiniones en coincidencia”. Revista Question, vol. 7. Extraída el 2/ IX/2015 desde http://perio.unlp.edu.ar/ojs/index.php/question/issue/view/18

_(2002). “Las campañas preventivas en torno al cólera y el SIDA”, pp. 429-432. En Morales, $\bar{F}$. y otros (2002). Psicología Social. Buenos Aires: Prentice Hall, Pearson Educacion. _(1992). “Las campañas... ¿De interés público?”, pp. 91-95. En Vacchieri, A. (comp.). El Medio es la TV. Buenos Aires: La Marca Editores.

Petracci, M. y Waisbord, S. (comps.) (2011). Comunicación y salud en la Argentina. Buenos Aires: La Crujía Ediciones.

Petracci, M.; Quevedo, L. y Vacchieri, A. (1998). "Los modelos político comunicativos del SIDA y el cólera en la Argentina, 1991-1993”. ZER Revista de Estudios de Comunicación, núm. 4, pp. 199-218.

Pulerwitz, J.; Barker, G. y Verma, R. (2012). "Changing Gender Norms for HIV and Violence Risk Reduction: A Comparison of Male-Focused Programs in Brazil and India", pp. 469-487. En Obregon, R. y Waisbord, S. (2012). The Handbook of Global Health Communication. Oxford: John Wiley \& Sons.

Ransohoff, D. y Ransohoff, R. (2001). "Sensacionalism in the media: when journalists and scientists may be complicit collaborators". Effective Clinical Practice, núm. 4, pp. 185-188.

Regis, S. (2007). "Reflexiones sobre las prácticas de la comunicación en el campo de la salud”. Revista Question, vol. 7, núm. 13. Extraída el 2/IX/2015 desde http://perio.unlp. edu.ar/ ojs/index.php/question/article/view/331 
Rodrigo Alsina, M. (1993). La construcción de la noticia. Barcelona: Paidós.

Román, V. (2011). "El rol del periodista ante la comunicación de enfermedades y su impacto", pp. 171-184. En Petracci, M. y Waisbord, S. (comps.). Comunicación y salud en la Argentina. Buenos Aires: La Crujía Ediciones.

Schvartz, D. (2011): “La comunicación médico-paciente”, pp. 201-226. En Petracci, M. y Waisbord, S. (comps.). Comunicación y salud en la Argentina. Buenos Aires: La Crujía Ediciones.

Schwarz, P. (2013). "Capacidad de agencia en salud a partir del uso de Nuevas Tecnologías de Información y Comunicación”. En Domínguez, A. (coord.). Salud Colectiva. Del padecimiento a la agencia social en salud colectiva. Personas que viven con enfermedades crónicas y vida cotidiana. Bariloche: Universidad de Río Negro.

Sengupta, A. y Elias, E. (2012). "Women's Health and Healing in the Peruvian Amazon: Minga's Perú's Participatory Communication Approach”, pp. 488-506. En Obregon, R. y Waisbord, S. (2012). The Handbook of Global Health Communication. Oxford: John Wiley \& Sons.

Vara, A. (2011). "El juego del miedo, el juego del estatus: promoción de una vacuna contra el HPV en la Argentina”, pp. 293-320. En Petracci, M. y Waisbord, S. (comps.). Comunicación y salud en la Argentina. Buenos Aires: La Crujía Ediciones.

Waisbord, S. (2010). "Cuando la salud es titular: dengue, gripe AH1N1 y ciclos mediáticos epidémicos”, pp. 185-200. En Petracci, M. y Waisbord, S. (comps.). Comunicación y salud en la Argentina. Buenos Aires: La Crujía Ediciones.

Webb, T. y otros (2010). "Using the Internet to Promote Health Behavior Change: A Systematic Review and Meta-analysis of the Impact of Theoretical Basis, Use of Behavior Change Techniques, and Mode of Delivery on Efficacy". Journal of Medical Internet Research, vol. 12, núm. 1. Extraída el 2/IX/2015 desde Disponible en: http://www.jmir. org/2010/1/e4/

Wolf, M. (2004). La investigación de la comunicación de masas. Buenos Aires: Paidós. 